\title{
Toward Chemistry in Real Space and Real Time
}

\section{Virtual Special Issue}

\begin{tabular}{|c|c|c|}
\hline $\begin{array}{l}\text { Toward Chemistry in Real Space and } \\
\text { Real Time Preface }\end{array}$ & $\begin{array}{l}\text { V. Ara Apkarian, Eric } \\
\text { Potma }\end{array}$ & 10.1021/acs.jpcc.0c02087 \\
\hline $\begin{array}{l}\text { Reversible Aggregation of } \\
\text { Covalently Cross-Linked Gold } \\
\text { Nanocrystals by Linker Oxidation }\end{array}$ & $\begin{array}{l}\text { Zhongyue Luan, Trenton } \\
\text { Salk, Alex Abelson, } \\
\text { Stephanie Jean, Matt Law }\end{array}$ & 10.1021/acs.jpcc.9b06976 \\
\hline $\begin{array}{l}\text { Tip-Enhanced Raman Nanographs of } \\
\text { Plasmonic Silver Nanoparticles }\end{array}$ & $\begin{array}{l}\text { Ashish Bhattarai, Irina V. } \\
\text { Novikova, Patrick Z. El- } \\
\text { Khoury }\end{array}$ & 10.1021/acs.jpcc.9b07811 \\
\hline $\begin{array}{l}\text { Flux-Conserving Diagrammatic } \\
\text { Formulation of Optical Spectroscopy } \\
\text { of Open Quantum Systems }\end{array}$ & $\begin{array}{l}\text { Shaul Mukamel, Michael } \\
\text { Galperin }\end{array}$ & 10.1021/acs.jpcc. 9 b08635 \\
\hline $\begin{array}{l}\text { SERS Study of the Mechanism of } \\
\text { Plasmon-Driven Hot Electron } \\
\text { Transfer between Gold } \\
\text { Nanoparticles and PCBM }\end{array}$ & $\begin{array}{l}\text { Yue Wu, Muwen Yang, } \\
\text { Tyler W. Ueltschi, Martín } \\
\text { A. Mosquera, Zhu Chen, } \\
\text { George C. Schatz, Richard } \\
\text { P. Van Duyne }\end{array}$ & 10.1021/acs.jpcc.9b10395 \\
\hline $\begin{array}{l}\text { Defining Multiple Configurations of } \\
\text { Rubrene on a Ag(100) Surface with } \\
5 \text { A Spatial Resolution via Ultrahigh } \\
\text { Vacuum Tip-Enhanced Raman } \\
\text { Spectroscopy }\end{array}$ & $\begin{array}{l}\text { Jeremy F. Schultz, Linfei } \\
\text { Li, Sayantan Mahapatra, } \\
\text { Chasen Shaw, Xu Zhang, } \\
\text { Nan Jiang }\end{array}$ & 10.1021/acs.jpcc. 9 b09162 \\
\hline $\begin{array}{l}\text { SERS Nanoprobe for Intracellular } \\
\text { Monitoring of Viral Mutations }\end{array}$ & $\begin{array}{l}\text { Kholud Dardir, Hao Wang, } \\
\text { Brigitte E. Martin, Maria } \\
\text { Atzampou, Christopher B. } \\
\text { Brooke, Laura Fabris }\end{array}$ & 10.1021/acs.jpcc.9b09253 \\
\hline $\begin{array}{l}\text { Orbital Control and Coherent Charge } \\
\text { Transport in Transition Metal } \\
\text { Platinum(II)-Platinum(II) Lantern } \\
\text { Complexes in Molecular Junctions }\end{array}$ & $\begin{array}{l}\text { Leighton O. Jones, Martín } \\
\text { A. Mosquera, Mark A. } \\
\text { Ratner, George C. Schatz }\end{array}$ & 10.1021/acs.jpcc. 9 b10093 \\
\hline $\begin{array}{l}\text { Analytical Approaches To Identify } \\
\text { Plasmon-like Excited States in Bare } \\
\text { and Ligand-Protected Metal } \\
\text { Nanoclusters }\end{array}$ & $\begin{array}{l}\text { Rebecca L. M. Gieseking, } \\
\text { Adam P. Ashwell, Mark A. } \\
\text { Ratner, George C. Schatz }\end{array}$ & 10.1021/acs.jpcc. 9 b10569 \\
\hline $\begin{array}{l}\text { Optical Activity from the Exciton } \\
\text { Aharonov-Bohm Effect: A Floquet } \\
\text { Engineering Approach }\end{array}$ & $\begin{array}{l}\text { Kai Schwennicke, Joel } \\
\text { Yuen-Zhou }\end{array}$ & $10.1021 /$ acs.jpcc. $9 \mathrm{~b} 10030$ \\
\hline
\end{tabular}




\begin{tabular}{|c|c|c|}
\hline $\begin{array}{l}\text { Making Permanent Optical Matter of } \\
\text { Plasmonic Nanoparticles by in Situ } \\
\text { Photopolymerization }\end{array}$ & $\begin{array}{l}\text { Zhenzhen Chen, Fan Nan, } \\
\text { Zijie Yan }\end{array}$ & 10.1021/acs.jpcc.9b10267 \\
\hline $\begin{array}{l}\text { Influence of the Chemical Structure } \\
\text { on Molecular Light Emission in } \\
\text { Strongly Localized Plasmonic Fields }\end{array}$ & $\begin{array}{l}\text { Yao Zhang, Zhen-Chao } \\
\text { Dong, Javier Aizpurua }\end{array}$ & 10.1021/acs.jpcc.9b10256 \\
\hline $\begin{array}{l}\text { Staphyloxanthin Photolysis } \\
\text { Potentiates Low Concentration } \\
\text { Silver Nanoparticles in Eradication } \\
\text { of Methicillin-Resistant } \\
\text { Staphylococcus aureus }\end{array}$ & $\begin{array}{l}\text { Sebastian Jusuf, Jie Hui, } \\
\text { Pu-Ting Dong, Ji-Xin } \\
\text { Cheng }\end{array}$ & 10.1021/acs.jpcc.9b10209 \\
\hline $\begin{array}{l}\text { Zinc Oxide @ Silica Core/Shell } \\
\text { Microspheres for Single-Molecule } \\
\text { Force Microscopy in Aqueous and } \\
\text { Nonaqueous Solvents }\end{array}$ & $\begin{array}{l}\text { Qixuan Yu, Ya-Na Chen, } \\
\text { Jacob W. Black, Ziad } \\
\text { Ganim }\end{array}$ & 10.1021/acs.jpcc.9b11923 \\
\hline $\begin{array}{l}\text { Uniting Top-Down and Bottom-Up } \\
\text { Strategies Using Fabricated } \\
\text { Nanostructures as Hosts for } \\
\text { Synthesis of Nanomites }\end{array}$ & $\begin{array}{l}\text { Cady A. Lancaster, Wallis } \\
\text { E. Scholl, Matthew A. } \\
\text { Ticknor, Jennifer S. } \\
\text { Shumaker-Parry }\end{array}$ & $10.1021 /$ acs.jpcc. $9 \mathrm{~b} 11820$ \\
\hline $\begin{array}{l}\text { Chemically Selective Imaging of } \\
\text { Individual Bonds through Scanning } \\
\text { Electron Energy-Loss Spectroscopy: } \\
\text { Disulfide Bridges Linking Gold } \\
\text { Nanoclusters }\end{array}$ & $\begin{array}{l}\text { Karolina Sokołowska, } \\
\text { Zhongyue Luan, Eero } \\
\text { Hulkko, Christoph } \\
\text { Rameshan, Noelia } \\
\text { Barrabés, Vartkess A. } \\
\text { Apkarian, Tanja Lahtinen }\end{array}$ & 10.1021/acs.jpclett.9b03496 \\
\hline
\end{tabular}

